\title{
TRATAMENTO DE ÁGUA CONTAMINADA COM FÁRMACOS POR MEIO DE FOTÓLISE UV E UV/H $\mathrm{H}_{2} \mathrm{O}_{2}$.
}

\author{
C. PEREIRA-KATSUMATA ${ }^{1}$, M. P. SILVA ${ }^{1}{ }^{1}$ A. P. S. BATISTA ${ }^{1}$ e A. C. S. C. TEIXEIRA ${ }^{1}$ \\ ${ }^{1}$ Universidade de São Paulo, Escola Politécnica, Departamento de Engenharia Química \\ E-mail para contato: percaroline@gmail.com
}

\begin{abstract}
RESUMO - Estudou-se a degradação dos fármacos acetaminofeno, atenolol, bezafibrato, diclofenaco e ibuprofeno em solução aquosa através de fotólise e peroxidação fotoassistida. Foram determinados os coeficientes de absorção molar e realizaram-se ensaios de hidrólise e caracterização das soluções antes e após os processos de fotólise e $\mathrm{UV} / \mathrm{H}_{2} \mathrm{O}_{2}$. As degradações foram realizadas em um reator anular com lâmpada de vapor de mercúrio de baixa pressão de $75 \mathrm{~W}$ e concentrações iniciais de 5 e $20 \mathrm{mg} / \mathrm{L}$ de cada fármaco. Os coeficientes de absorção molar mostraram-se muito dependentes do $\mathrm{pH}$ e não houve degradação por hidrólise após $24 \mathrm{~h}$. O diclofenaco e o bezafibrato degradaram-se mais rapidamente, independentemente do processo foto-assistido usado; a degradação ocorreu mais rapidamente quanto menor a concentração inicial dos fármacos e na presença de peróxido. Esses resultados são úteis para compreensão da remediação de águas contendo esses contaminantes emergentes por meio de processos foto-oxidativos.
\end{abstract}

\section{INTRODUÇÃO}

Estudos relataram que tem sido detectada grande variedade de fármacos e metabólitos em corpos d'água. Estes micropoluentes começaram a chamar a atenção da comunidade científica, pois as estações convencionais de tratamento de água e efluentes, salvo em alguns casos, não têm processos de tratamento adequados para degradá-los, eliminá-los ou removê-los completamente da matriz aquosa (SILVA e COLLINS, 2011). Os processos de oxidação avançada (POA), fotoquímicos ou não fotoquímicos, são uma alternativa para a degradação desses poluentes emergentes. Nesses processos há geração de radicais hidroxila $(\bullet \mathrm{OH})$, espécies de forte caráter oxidante, que reagem com a maioria dos compostos orgânicos, com baixa seletividade e reações de segunda ordem (constantes cinéticas da ordem de 108-1010 L mol-1 s-1 (OPPENLÄNDER, 2003). Neste trabalho é estudada a degradação dos fármacos acetaminofeno (ACT), atenolol (ATL), bezafibrato (BZF), diclofenaco (DIC) e ibuprofeno (IBU), identificados no Reservatório Billings, segundo Almeida e Weber (2005). A degradação dos fármacos é realizada por meio dos processos de fotólise UV e peroxidação fotoassistida $\left(\mathrm{UV} / \mathrm{H}_{2} \mathrm{O}_{2}\right)$, caracterizando as soluções aquosas antes e após o tratamento quanto à degradação do fármaco, à remoção de carbono orgânico total e à toxicidade, a fim de avaliar o desempenho eficiência dos processos estudados.

\section{MATERIAIS E MÉTODOS}




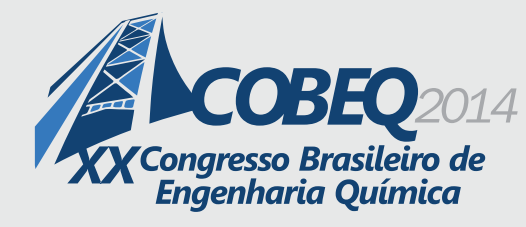

19 a 22 de outubro de 2014

Florianópolis/SC

Neste trabalho utilizaram-se fármacos de padrão analítico e foram adquiridos da SigmaAldrich. As soluções foram preparadas em água purificada por osmose inversa. Experimentos foram realizados com cada fármaco individualmente e também com a mistura entre eles. Optou-se por realizar o estudo com a mistura dos fármacos selecionados, pois é dessa forma que eles se encontram no meio real. Os valores de pKa (a constante de equilíbrio ácido-base) de cada substância foi determinada a partir de dez soluções aquosas de mesma concentração $\left(5 \mathrm{mg} \mathrm{L}^{-1}\right)$ com pH entre 2 e 11 , para as quais foram obtidos os espectros de absorção UV-visível empregando-se um espectrofotômetro Varian Cary 50. O pKa foi obtido a partir do gráfico de absorbância em função do $\mathrm{pH}$ para os comprimentos de onda em que se verificaram os máximos correspondentes às formas protonada e desprotonada de cada fármaco. Os coeficientes de absorção molar $(\varepsilon)$ de cada fármaco em função do comprimento de onda, em pH 2, 5 e 12, foram obtidos espectrofotometricamente a partir de soluções dos fármacos com concentrações de $0,05,1,2,3$ e $5 \mathrm{mg} \mathrm{L}^{-1}$.

Nos ensaios de hidrólise, em todos os casos a concentração nominal inicial do fármaco foi 5 $\mathrm{mg} \mathrm{L}^{-1}$. Realizaram-se esses ensaios em três diferentes valores de $\mathrm{pH}$ (3, 7 e 9). O ensaio foi realizado a $25^{\circ} \mathrm{C}$, em frascos âmbar. Os experimentos foram realizados em triplicata, as amostras coletadas nos tempos 0,6 e $24 \mathrm{~h}$ e analisadas por HPLC.

Os ensaios de fotólise foram realizadas com a solução aquosa da mistura dos cinco fármacos, em um reator fotoquímico anular de vidro (volume irradiado de 3,9 L). A irradiação foi feita por uma lâmpada de vapor de mercúrio de baixa pressão (emissão em $254 \mathrm{~nm}$ ) com potência de $75 \mathrm{~W}$ posicionada concentricamente no reator. Utilizaram-se $5 \mathrm{~L}$ de solução aquosa dos fármacos $(5$ ou 20 $\mathrm{mg} \mathrm{L}^{-1}$ de cada fármaco). A solução foi mantida em $\mathrm{pH} \leq 2$. Os experimentos de degradação pelo processo $\mathrm{UV} / \mathrm{H}_{2} \mathrm{O}_{2}$ foram realizadas de forma análoga aos experimentos de fotólise, com adição da solução de $\mathrm{H}_{2} \mathrm{O}_{2}$ concentrada ( $30 \%$ em massa) em solução aquosa, previamente à irradiação. $\mathrm{O}$ cálculo do volume de solução de $\mathrm{H}_{2} \mathrm{O}_{2} 30 \%$ a ser utilizada foi realizado considerando a quantidade necessária para mineralização total de cada fármaco, estimada com base na estequiometria das reações. Foram realizados experimentos com a mistura dos fármacos com concentração inicial de cada de 5 ou $20 \mathrm{mg} \mathrm{L}^{-1}$ e potência da lâmpada de $75 \mathrm{~W}$. A concentração de cada fármaco foi quantificada por cromatografia líquida de alta eficiência (HPLC), empregando-se um equipamento Shimadzu (modelo 10 AD) equipado com um detector UV (modelo SPD-20A). Fez-se necessária a otimização da análise cromatográfica, para analisar simultaneamente os fármacos escolhidos. $\mathrm{O}$ gradiente de eluição está descrito na Tabela 1 . O volume de injeção utilizado foi $25 \mu \mathrm{L}$, vazão de 1

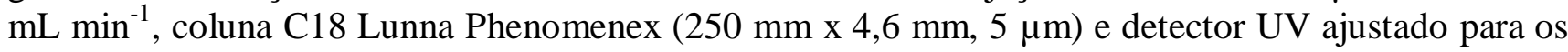
comprimentos de onda 225 e $254 \mathrm{~nm}$.

A concentração de carbono orgânico total (TOC) foi determinada em um equipamento Shimadzu (modelo 5000A).

Os ensaios de toxicidade aguda foram realizados para amostras com 0, 15, 45 e 120 minutos de tratamento por fotólise empregando-se a bactéria Vibrio fischeri em um equipamento Microtox ${ }^{\circledR}$ 500, conforme a norma CETESB/L.5227/dez/2001. 
Tabela 1 - Gradiente de eluição para os componentes da fase móvel, empregado na análise dos fármacos por HPLC.

\begin{tabular}{|c|c|c|}
\hline Tempo (min) & Fase $\mathbf{A}^{\mathbf{a}}(\%)$ & Fase $^{b}{ }^{b}(\%)$ \\
\hline 0,01 & 90 & 10 \\
\hline 7,0 & 90 & 10 \\
\hline 10,0 & 20 & 80 \\
\hline 30,0 & 20 & 80 \\
\hline 32,0 & 90 & 10 \\
\hline 35,0 & 90 & 10 \\
\hline
\end{tabular}

${ }^{\mathrm{a}}$ Fase A: Água $+0,2 \%$ ácido acético ${ }^{\mathrm{b}}$ Fase B: Metanol + 0,2\% ácido acético

Para determinar a concentração residual de $\mathrm{H}_{2} \mathrm{O}_{2}$ nos experimentos $\mathrm{UV} / \mathrm{H}_{2} \mathrm{O}_{2}$ utilizou-se o método descrita por Nogueira et al. (2005), segundo o qual faz-se reagir o $\mathrm{H}_{2} \mathrm{O}_{2}$ residual com metavanadato de amônio $\left(\mathrm{NH}_{4} \mathrm{VO}_{3}\right)$, que tem como produto de reação o íon peroxovanádio. Para a análise, foram misturados $1,0 \mathrm{~mL}$ (para o ensaio de concentração inicial dos fármacos $5 \mathrm{mg} \mathrm{L}^{-1}$ ) ou $5,0 \mathrm{~mL}$ (para o ensaio de concentração inicial $20 \mathrm{mg} \mathrm{L}^{-1}$ ) de amostra a $1,0 \mathrm{~mL}$ de solução de $\mathrm{NH}_{4} \mathrm{VO}_{3}$, completando-se o volume com água até $10,0 \mathrm{~mL}$. A quantificação do íon peroxovanádio é então determinada espectrofotometricamente a partir da medida da absorbância da solução em $450 \mathrm{~nm}$ empregando-se um espectrofotômetro UV-visível (Varian Cary 50).

\section{RESULTADOS E DISCUSSÃO}

A Figura 1 compara os valores de $\varepsilon$ em $254 \mathrm{~nm}$ para cada fármaco, em alguns casos muito dependentes do $\mathrm{pH}$. Para os fármacos ATL, BZF, DIC e IBU, a variação dos valores é pequena, porém para o ACT há um deslocamento importante na curva em pH 12 para comprimentos de onda maiores (red shift). A absorção da radiação UV $(254 \mathrm{~nm})$ pelo ACT é superior à dos demais fármacos $\left(\varepsilon=8990 \mathrm{~L} \mathrm{~mol}^{-1} \mathrm{~cm}^{-1}\right.$ ), enquanto a absorção pelo ATL e pelo IBU é comparativamente muito inferior ( $\varepsilon=725$ e $1080 \mathrm{~mol}^{-1} \mathrm{~cm}^{-1}$, respectivamente). Os valores de $\varepsilon$ mostram ainda que os fármacos competem pela absorção da radiação incidente em 254 nm (OPPENLÄNDER, 2003).

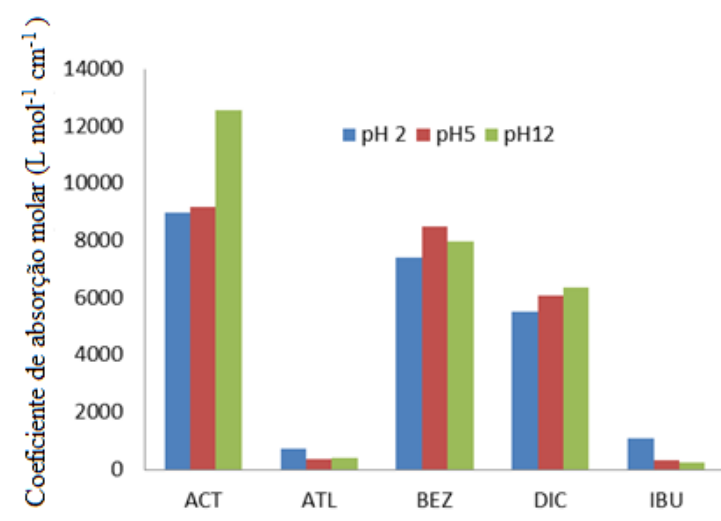

Figura 1 - Coeficientes de absorção molar dos fármacos estudados em $254 \mathrm{~nm}$ a diferentes pH. 


\section{9 a 22 de outubro de 2014 \\ Florianópolis/SC}

Os pKa medidos foram 9,0 (ACT), 9,3 (ATL), 3,5 (BZF), 4,0 (DIC) e 4,7 (IBU), muito próximos dos valores da literatura, como apresentados por SERRANO et al. (2011), KUJAWAROELEVELD et al. (2008), LORPHENSRI et al. (2006), MARTÍNEZ et al. (2000); esses resultados indicam que todos os fármacos estão na sua forma neutra nos experimentos de fotólise $(\mathrm{pH} \leq 2)$, à exceção do ATL.

Os experimentos de hidrólise comprovam que na ausência de luz, durante 24 horas, independentemente dos valores de $\mathrm{pH}$, as soluções apresentaram estabilidade, não sendo observada hidrólise dos fármacos individuais, nem na mistura. A implicação importante para essa conclusão para o ponto de vista dos experimentos é que a degradação dos fármacos em estudos por fotólise tem como único fator a interação da radiação UV com os fármacos. Em ponto de vista ambiental é importante ressaltar sua persistência em ambientes aquáticos.

$\mathrm{O}$ experimento de fotólise com a solução dos fármacos foi realizado em $\mathrm{pH} 2$, empregando lâmpadas de $75 \mathrm{~W}$ e concentrações iniciais de cada fármaco de 5 e $20 \mathrm{mg} \mathrm{L}^{-1}$. Os gráficos de concentração e TOC (normalizados) em função do tempo, para os experimentos de fotólise sob radiação UV (254 nm), são apresentados na Figura 2.
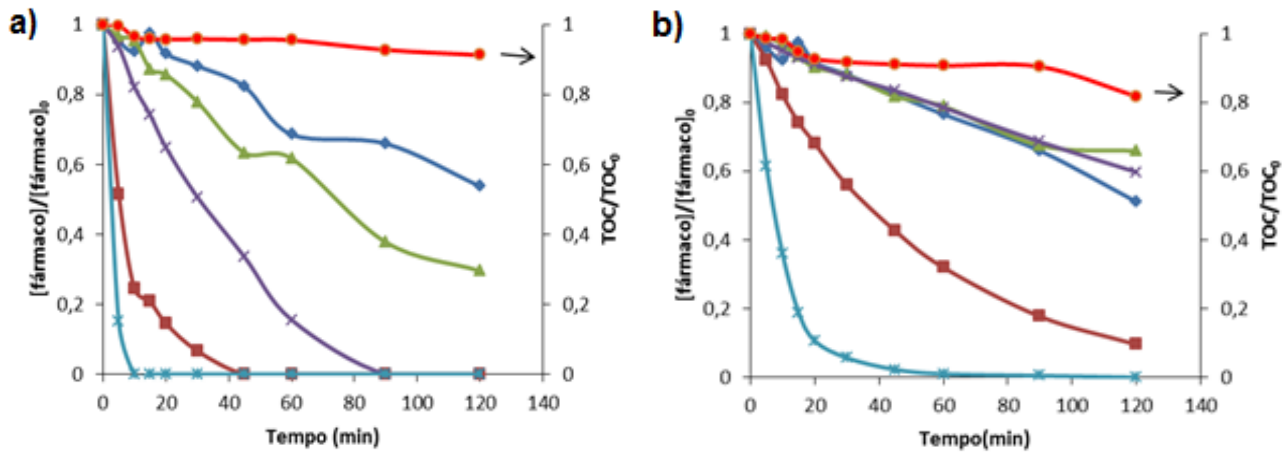

Figura 2 - Resultados dos experimentos de fotólise dos fármacos estudados. (a) $5 \mathrm{mg} \mathrm{L}^{-1}$ e $75 \mathrm{~W}$;

(b) $20 \mathrm{mg} \mathrm{L}^{-1}$ e $75 \mathrm{~W}$. A concentração indicada refere-se à concentração inicial nominal de cada fármaco na solução aquosa. $(\rightarrow$ ATL $\rightarrow$ ACT $\rightarrow$ BZF $*$ DIC $\rightarrow$ BBU $\rightarrow$ TOC $)$.

Os resultados da Figura 2 mostram que as velocidades de degradação dos fármacos diminuem com o aumento da concentração inicial. A fotólise permite degradar facilmente o DIC e o BZF, mas para os outros fármacos há necessidade de maior tempo de exposição. O ATL apresenta o menor valor do coeficiente de absorção molar $\varepsilon$ (Figura 1), o que está de acordo com a menor degradação por fotólise; resultado similar foi encontrado por Salgado et al., 2013. Por outro lado, a degradação do ACT foi mais lenta que a do DIC e a do BZF, particularmente para maior concentração inicial, o que leva a supor que a fotólise do ACT ocorra com menor rendimento quântico. Para o IBU obteve-se comportamento semelhante em todos os experimentos e é necessário maior tempo de exposição para degradá-lo completamente. $\mathrm{Na}$ Tabela 2, estão apresentados os resultados da análise dos dados de concentração em função do tempo segundo modelos de ordem zero ou de pseudo primeira-ordem (constante de velocidade, coeficiente de determinação, $\mathrm{R}^{2}$, tempo de meia vida e a porcentagem de remoção em $120 \mathrm{~min}$ ) para os experimentos: (A) $5 \mathrm{mg} \mathrm{L}^{-1}$ e $75 \mathrm{~W}$; (B) $20 \mathrm{mg} \mathrm{L}^{-1}$ e $75 \mathrm{~W}$. Analisando- 
se os resultados apresentados na Tabela 2 para o ACT, pode-se observar que quando o produto $\varepsilon[\mathrm{ACT}]$ em $\mathrm{t}=0$ é baixo (Figura $2 \mathrm{a}$ ) a concentração do fármaco diminuiu seguindo aproximadamente um comportamento de pseudo primeira-ordem e que quando o produto $\varepsilon[\mathrm{ACT}]$ em $\mathrm{t}=0$ é alto (Figura b), o comportamento da concentração com o tempo foi de ordem zero.

OPPENLÄNDER (2003) discute o efeito da concentração inicial e do coeficiente de absorção molar sobre a evolução da concentração do composto durante a fotólise em sistemas batelada, o que pode ser aplicado para o caso da degradação o ACT. Como apresentado pelo autor, o balanço molar para um composto $M$ que sofre fotólise em solução aquosa em um sistema batelada exposto à radiação monocromática resulta na Equação 1, em que $\Phi_{M}$ é o rendimento quântico da fotólise de $M$, $E_{p_{0} 0}$ é o fluxo fotônico incidente (mantido constante) A é a área irradiada, V é o volume irradiado, $l$ é o caminho óptico e $\mathrm{N}_{\mathrm{a}}$, a constante de Avogadro.

$$
-\frac{d[M]}{d t}=\Phi_{M} \cdot E_{P_{s} 0} \cdot\left(1-10^{-z_{\lambda}^{M} \cdot[M] l}\right) \frac{A}{N a \cdot V}
$$

Tabela 2 - Modelos de decaimento da concentração dos fármacos, coeficiente de determinação $\left(\mathrm{R}^{2}\right)$, constante de velocidade $(\mathrm{k})$, tempo de meia vida $\left(\mathrm{t}_{1 / 2}\right)$ e porcentagem de remoção em 120 min para os experimentos: (A) $5 \mathrm{mg} \mathrm{L}^{-1}$ e $75 \mathrm{~W}$; (B) $20 \mathrm{mg} \mathrm{L}^{-1}$ e $75 \mathrm{~W}$.

\begin{tabular}{|c|c|c|c|c|c|}
\hline Composto & Modelo & $\mathbf{R}^{2}$ & Constante $(\mathbf{k})^{\mathrm{a}}$ & $\begin{array}{c}\mathbf{t}_{1 / 2} \\
(\mathbf{m i n}) \\
\end{array}$ & $\begin{array}{c}\text { \% Remoção } \\
\text { (120 min) }\end{array}$ \\
\hline \multicolumn{6}{|c|}{$\mathbf{A}$} \\
\hline Acetaminofeno & {$[\mathrm{ACT}]=1,12 \mathrm{e}^{-0,03 \mathrm{t}}$} & 0,971 & 0,03 & 30 & 99 \\
\hline Atenolol & {$[\mathrm{ATL}]=1,0033 \mathrm{e}^{-0,005 \mathrm{t}}$} & 0,960 & 0,005 & 125 & 40 \\
\hline Bezafibrato & {$[\mathrm{BZF}]=0,7961 \mathrm{e}^{-0,086 \mathrm{t}}$} & 0,963 & 0,086 & 8,1 & 99 \\
\hline Diclofenaco & {$[\mathrm{DIC}]=1,0882 \mathrm{e}^{-0,189 t}$} & 0,977 & 0,189 & 3,66 & 99 \\
\hline Ibuprofeno & {$[\mathrm{IBU}]=1,0388 \mathrm{e}^{-0,01 \mathrm{t}}$} & 0,987 & 0,01 & 74 & 71 \\
\hline \multicolumn{6}{|c|}{ 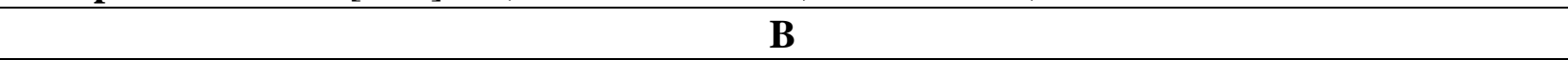 } \\
\hline Acetaminofeno & {$[\mathrm{ACT}]=-0,0033 \mathrm{t}+0,9847$} & 0,996 & 0,0033 & 130 & 40 \\
\hline Atenolol & {$[\mathrm{ATL}]=1,0159 \mathrm{e}^{-0,005 \mathrm{t}}$} & 0,972 & 0,005 & 138,63 & 49 \\
\hline Bezafibrato & {$[\mathrm{BZF}]=1,0067 \mathrm{e}^{-0,019 \mathrm{t}}$} & 0,999 & 0,019 & 36,5 & 90 \\
\hline Diclofenaco & {$[\mathrm{DIC}]=0,7282 \mathrm{e}^{-0,076 \mathrm{t}}$} & 0,971 & 0,076 & 9,1 & 99 \\
\hline Ibuprofeno & {$[\mathrm{IBU}]=0,9897 \mathrm{e}^{-0,004 \mathrm{t}}$} & 0,972 & 0,004 & 173,28 & 34 \\
\hline
\end{tabular}

${ }^{\mathrm{a}}$ Para pseudo ordem zero, $[\mathrm{k}]=\mathrm{mg} \mathrm{L}^{-1} \mathrm{~min}^{-1}$; para pseudo primeira-ordem, $[\mathrm{k}]=\mathrm{min}^{-1}$.

Quando a absorção de radiação pelo composto $\mathrm{M}$ for alta, devido ao fato do valor coeficiente de absorção molar a um determinado comprimento de onda for grande e/ou a concentração de $\mathrm{M}$ for alta $A_{\lambda}=\varepsilon_{\lambda}^{M} \cdot[M] l>2$ ) então o termo $10^{-\varepsilon_{\alpha}^{M}{ }^{M}[M] l}$ torna-se próximo de zero. Nesse caso, a integração da Equação 1 resulta:

$$
[M]_{t}=[M]_{0}-\Phi_{M} \cdot E_{P, 0} \times \frac{A}{N a \cdot V} \times t
$$


Portanto o processo de degradação de M por fotólise segue cinética de pseudo ordem zero.

Quando a absorção for baixa $\left(A_{\lambda}=\varepsilon_{\lambda^{M}}^{M} \cdot[M] l \ll 1\right)$, então:

$$
10^{-\varepsilon_{2}^{M} \cdot[M] l} \cong 2,303 \varepsilon_{\lambda^{M}}^{M}[M] l \text {. }
$$

Nesse caso, a integração da Equação (1) fica:

$$
[M]_{t}=[M]_{0} \exp \left[-\left(\Phi_{M} \cdot E_{P_{s} 0} \cdot 2,303 \varepsilon_{\Omega_{2}}^{M}[M] l \cdot \frac{A}{N a \cdot V}\right) t\right]
$$

A qual corresponde a um modelo cinético de pseudo primeira-ordem.

No caso da mistura de fármacos, o lado direito da Equação (1) deve ser multiplicada pelo fator $\varepsilon .[\mathrm{M}] / \Sigma \varepsilon$. [M], que representa a fração da radiação incidente absorvida pelo fármaco em relação à absorção pela mistura. Assim, o emprego desse modelo deve ser analisado com cautela, porque pode haver formação de subprodutos de degradação que também interferem na absorção de radiação UV. Para os fármacos ATL, BZF, DIC e IBU, não ocorrerem mudanças nos comportamentos das taxas de degradação com a variação da concentração inicial.

Por sua vez, os valores de TOC mostraram um pequeno decréscimo em todos os experimentos ( $13 \%$ ao final de 120 minutos), o que comprova a persistência dos produtos de degradação formados.

Na Tabela 3 são apresentados os resultados dos ensaios de toxicidade obtidos com a bactéria Vibrio fischeri, para as amostras retiradas dos experimentos de degradação por fotólise com a mistura de fármacos. Observa-se que os valores de $\mathrm{CE}_{50}$ seguiram o mesmo comportamento em todos os experimentos, diminuindo a partir do valor inicial até 15 minutos, aumentando em 45 minutos e diminuindo novamente após 120 minutos de tratamento. A classificação proposta por Tonkes et al. (1999) considera que as amostras com um valor de $\mathrm{CE}_{50}$ inferior a $10 \%$ são consideradas tóxicas e amostras com um valor de $\mathrm{CE}_{50}$ inferior a $1 \%$ como muito tóxicas.

Tabela 3 - Valores de $\mathrm{CE}_{50}(\%)$ nos tempos de 0, 15, 45 e 120 min de degradação por fotólise da solução de mistura de fármacos. ${ }^{a}$

\begin{tabular}{ccccc}
\hline Experimentos $^{\mathbf{b}}$ & $\mathbf{0} \mathbf{m i n}$ & $\mathbf{1 5} \mathbf{~}$ in & $\mathbf{4 5} \mathbf{~ m i n}$ & $\mathbf{1 2 0} \mathbf{~ m i n}$ \\
\hline $5 \mathrm{mg} \mathrm{L}^{-1} / 75 \mathrm{~W}$ & 1,4 & 0,9 & 1,6 & 0,5 \\
$20 \mathrm{mg} \mathrm{L}^{-1} / 75 \mathrm{~W}$ & 2,3 & 1,3 & 2,0 & 1,2 \\
\hline
\end{tabular}

${ }^{a}$ Os valores $\mathrm{CE}_{50}$ indicam a \% da amostra que exerce efeito deletério a 50\% dos organismo-teste.

${ }^{\mathrm{b}}$ A concentração refere-se à concentração inicial nominal de cada fármaco na solução.

Em todos os experimentos de fotólise as amostras tornaram-se mais tóxicas frente ao organismo-teste após irradiação UV prolongada, apesar de em alguns casos ocorrer degradação rápida e praticamente total de alguns fármacos, obtendo-se concentrações abaixo do limite de quantificação 


\section{9 a 22 de outubro de 2014 \\ Florianópolis/SC}

cromatográfico. As taxas de remoção de TOC muito baixas obtidas nos experimentos estão associadas à formação de produtos de degradação persistentes e de maior toxicidade.

Os históricos de concentração em função do tempo nos experimentos de $\mathrm{UV} / \mathrm{H}_{2} \mathrm{O}_{2}$ sob radiação UV (254 nm) são apresentadas na Figura 3, para mistura dos fármacos com concentração de $5 \mathrm{mg} \mathrm{L}^{-1}$ de cada e concentração de $3,6 \mathrm{mmol} \mathrm{L}^{-1}$ de peróxido (experimento I) e para mistura dos fármacos com concentração de $20 \mathrm{mg} \mathrm{L}^{-1}$ cada e concentração de $14,45 \mathrm{mmol} \mathrm{L}^{-1}$ de peróxido (experimento II). Comparando os gráficos da Figura 2 e 3, observa-se que a influência do $\mathrm{H}_{2} \mathrm{O}_{2}$ corresponde a um aumento da velocidade de degradação dos fármacos em relação ao processo de fotólise apenas. As concentrações dos fármacos em estudo estavam abaixo do limite de detecção após 20 min para todos os fármacos no experimento I e após 50 min no experimento II, indicando que quanto menor a concentração dos fármacos, mais rápida ocorre a degradação, afirmando que a concentração de peróxido sejam proporcionais as concentrações dos fármacos. O monitoramento da concentração de $\mathrm{H}_{2} \mathrm{O}_{2}$ indicou praticamente seu total consumo no experimento I; já no experimento II não foi alcançado o total consumo de $\mathrm{H}_{2} \mathrm{O}_{2}$, porém obteve uma redução da concentração de aproximadamente $81,4 \%$.
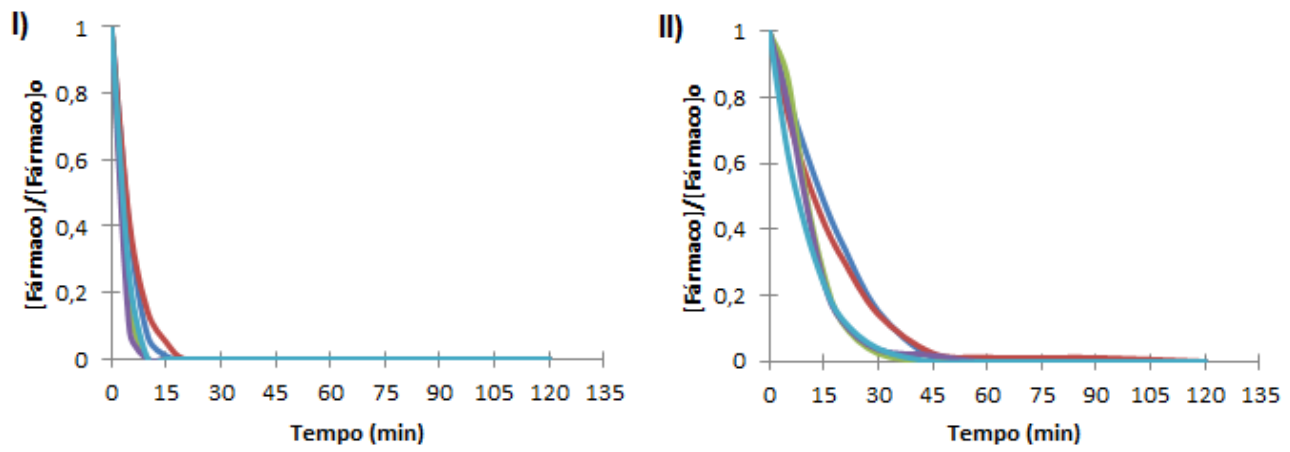

Figura 3 - Resultado do experimento de $\mathrm{UV} / \mathrm{H}_{2} \mathrm{O}_{2}$ dos fármacos. (I) $5 \mathrm{mg} \mathrm{L}^{-1}, 75 \mathrm{~W}$ e concentração de $3,6 \mathrm{mmol} \mathrm{L}^{-1}$ de $\mathrm{H}_{2} \mathrm{O}_{2}$ e (II) $20 \mathrm{mg} \mathrm{L}^{-1}$,75 W e concentração de $14,45 \mathrm{mmol} \mathrm{L}^{-1}$ de $\mathrm{H}_{2} \mathrm{O}_{2}$. A concentração indicada refere-se à concentração inicial nominal de cada fármaco na solução aquosa (-ACT-ATL - BZF - DIC - IBU).

\section{CONCLUSÕES}

Os fármacos estudados não hidrolisaram após $24 \mathrm{~h}$, em diferentes $\mathrm{pH}$, o que indica sua estabilidade na ausência de radiação UV-visível. O DIC e o BZF são os fármacos que degradam mais rapidamente por $\mathrm{UV}$ e $\mathrm{UV} / \mathrm{H}_{2} \mathrm{O}_{2}$, cuja velocidade é tão maior quanto menor a concentração inicial dos fármacos. A degradação do ATL foi a mais lenta, provavelmente devido à baixa absorção da radiação por este fármaco, que possui o menor valor do $\varepsilon$ encontrado. Por outro lado, os resultados sugerem que a fotólise do ACT ocorre com rendimento quântico muito baixo. A fotólise da mistura resulta, no entanto, em produtos de degradação persistentes, com pequena remoção do TOC e aumento de toxicidade frente ao organismo-teste Vibrio fischeri, após 120 minutos. Conclui-se também que a presença de $\mathrm{H}_{2} \mathrm{O}_{2}$ mostra efeito positivo quanto ao aumento da velocidade de degradação dos

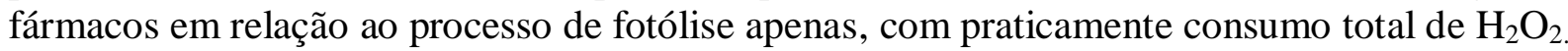




\section{REFERÊNCIAS}

ALMEIDA, G. A.; WEBER, R. R. Fármacos na Represa Billings. Rev. Saúde Amb., v. 6, p. 7-13, 2005.

COMPANHIA DE TECNOLOGIA DE SANEAMENTO AMBIENTAL L.5.227: Teste de toxicidade com a bactéria luminescente Vibrio Fischeri (Método de Ensaio). São Paulo, 2001.

KUJAWA-ROELEVELD, K.; SCHUMAN, E.; GROTENHUIS, T.; KRAGIĆ, D.; MELS, A.; ZEEMAN, G. Biodegradability of human pharmaceutically active compounds (PhAC) in biological systems treating source separated wastewater streams. In: Third SWITCH Scientific Meeting Belo Horizonte, Brazil, 2008.

LORPHENSRI, O; INTRAVIJIT, J.; SABATINI, D. A.; KIBBEY, T.C.G.; OSATHAPHAN, K.; SAIWAN. C. Sorption of acetaminophen, 17 $\alpha$-ethynyl estradiol, nalidixic acid, and norfloxacin to silica, alumina, and a hydrophobic medium. Water Res., v. 40, p. 1481-1491, 2006.

MARTÍNEZ, V.; MAGUREGUI, M. I.; JIMÉNEZ, R. M.; ALONSO, R. M. Determination of the pKa values of beta-blockers by automated potentiometric titrations. J. Pharm. Biomed. Anal., v. 23, p. 459-468, 2000.

NOGUEIRA, R. F. P.; SILVA, M. R. A.; TROVÓ, A. G. Influence of the iron source on the solar photo-Fenton degradation of different classes of organic compounds. Solar Energy,v.79, p. 384392, 2005.

OPPENLÄNDER, T. Photochemical Purification of Water and Air: Advanced Oxidation Processes (AOPS): Principles, Reaction Mechanisms, Reactor Concepts, Wiley-VCH, 2003.

SALGADO, R.; PEREIRA, V. J.; CARVALHO, G.; SOEIRO, R.; GAFFNEY, V.; ALMEIDA, C.; CARDOSO, V.; FERREIRA, E.; BENOLIEL, M. J.; TERNES, T. A.; OEHMEN, A.; REIS, M. A. M.; NORONHA, J. P. Photodegradation kinetics and transformation products of ketoprofen, diclofenac and atenolol in pure water and treated wastewater. J. Haz. Mat., v. 244, p. 516-527, 2013.

SERRANO, D.; SUÁREZ, S.; LEMA, J. M.; OMIL, F. Removal of persistent pharmaceutical micropollutants from sewage by addition of PAC in a sequential membrane bioreactor. Water Res., v. 45, p. 5323-5333, 2011.

SILVA, C. G. A.; COLLINS, C. H. Aplicações de cromatografia líquida de alta eficiência para o estudo de poluentes orgânicos emergentes. Quím. Nova, v. 34, p. 665-676, 2011.

TONKES, M.; GRAAF, P. J. F.; GRAANSMA, J. Assessment of complex industrial effluents in the Netherlands using a whole effluent toxicity (or wet) approach. Water Sci. Technol., v. 39, p. 55-62, 1999. 